\title{
Deep Vein Thrombosis in Elderly Patients following Surgery for Fracture of the Proximal Femur
}

\author{
R Magetsari, PhD, P Dewo, PhD, AS Nugroho, MD, Z Lanodiyu, MD \\ Department of Orthopaedics and Traumatology, Sardjito General Hospital/Universitas Gadjah Mada, \\ Yogyakarta, Indonesia
}

\begin{abstract}
The incidence of Deep Vein Thrombosis (DVT) is high in orthopaedic cases, associated with morbidity and mortality. This study was done to evaluate the incidence of DVT and factors that contributed to DVT in elderly patients with proximal femur fracture who underwent surgery. Patients 60 years of age and older with proximal femur fracture who underwent surgery were enrolled into this study. Daily observation of the clinical signs of DVT was done post operatively until the subjects were discharged from the hospital. D-Dimer test and contrast venography were carried out on Day 2 and between Days 4 and 7 after surgery. Results were interpreted as normal and obstruction. There were 42 eligible patients, 18 of them $(42.86 \%)$ were diagnosed with DVT. We found that positive D-Dimer test (1.40), longer duration of surgery (1.86), and presence of clinical symptoms (1.09) increased the risk of the occurence of thrombosis although not statistically significant. There were four DVT patients who passed away within 4 months after surgery due to complication from DVT. The incidence of DVT in elderly with proximal femur fracture underwent surgery was relatively high $(42.86 \%)$ with the mortality rate was $22 \%$.
\end{abstract}

Key Words:

Deep Vein Thrombosis; Proximal Femur Fracture

\section{INTRODUCTION}

Venous thromboembolism (VTE) refers to Deep Vein Thrombosis (DVT) and Pulmonary Embolism (PE). DVT is associated with relatively high mortality and morbidity. Within one year after the diagnosis, the mortality rate is about 20\% ${ }^{1}$. The incidence of DVT in Asian patients with hip fractures who had undergone total hip or total knee replacements without prophylaxis is approximately $17-58 \%$. These results were similiar to those previously reported in European population ${ }^{2,3}$. Untreated DVT can result in potentially fatal outcome, such as pulmonary embolism. In the US, the annual cost associated with diagnosis and treatment of VTE was estimated to be up to $\$ 15.5$ billion $^{4}$.
The number of proximal femur fractures is relatively high among elderly and the with increased indications for surgical interventions ${ }^{5,6}$. Early surgery and rehabilitation are expected to relieve pain, improve mobility, and reduce post-traumatic complications. When surgery is indicated, caution should be taken due to the prevemt potential risks of complications of thromboembolim post- operatively, especially in the elderly ${ }^{5,6}$. When comparing proximal and distal lower extremity fractures, the incidence of DVT is higher in the proximal part?

The outcome of DVT varies from complete resolution without any sequele to death due to pulmonary embolism ${ }^{8}$. Therefore, the need for its diagnosis and treatment has been growing rapidly in the last few decades. The incidence correlates with older age, longer operating times, and longer waiting period before fracture fixation ${ }^{7}$. The diagnosis of DVT cannot be made solely on its clinical presentation. Although pain and swelling are common presenting complaints, various signs and symptoms are used only to categorize the patients as having low, moderate, or high probability of $\mathrm{DVT}^{9}$. Diagnosis of DVT itself is made by contrast venography which has high sensitivity and specificity ${ }^{10}$. It is a radiographic study using contrast media injection via peripheral intravenous access. Although it is considered as an invasive test with defined risks such as contrast allergy and /or infection, it is a valuable and informative procedure for evaluating disorders of the venous system $^{11}$. The combination of informations obtained by contrast venography, clinical signs and non-invasive supporting examinations are used to diagnose, plan, and evaluate DVT especially in orthopaedic cases postoperatively.

Table I: Subject's Characteristics

\begin{tabular}{|lcc|}
\hline $\mathbf{N}=\mathbf{4 2}$ & \multicolumn{2}{c|}{ Venography } \\
& Normal & Thrombosis \\
\hline Age (mean) & 68.4 & 68.1 \\
Sex & 5 & 3 \\
$\quad$ Male & 19 & 15 \\
$\quad$ Female & & \\
\hline
\end{tabular}


Table II: Factors affecting the occurence of thrombosis

\begin{tabular}{|c|c|c|c|c|}
\hline & \multicolumn{2}{|c|}{ Venography } & \multirow[t]{2}{*}{ OR $95 \% \mathrm{Cl}$} & \multirow[t]{2}{*}{$P$ value } \\
\hline & Normal (n) & Thrombosis (n) & & \\
\hline \multicolumn{5}{|l|}{ D-Dimer } \\
\hline$<500$ & 14 & 9 & 1.40 & 0.591 \\
\hline$>500$ & 10 & 9 & $(0.34-5.75)$ & \\
\hline \multicolumn{5}{|l|}{ Duration of surgery } \\
\hline$<2$ hours & 13 & 7 & 1.86 & 0.327 \\
\hline$>2$ hours & 11 & 11 & $(0.45-7.79)$ & \\
\hline \multicolumn{5}{|l|}{ Clinical symptoms } \\
\hline Asymptomatic & 19 & 14 & 1.09 & 0.602 \\
\hline Symptomatic & 5 & 4 & $(0.19-5.96)$ & \\
\hline
\end{tabular}

D-Dimer is formed by the sequential action of thrombin, factor XIIIa, and plasmin. It is a unique marker of fibrin degradation that is useful in some cases, especially for the exclusion of VTE. The use of D Dimer test in DVT remains controversial. Several studies have shown the correlation of high D-Dimer with the incidence of DVT, but others have shown opposite results in hospitalized patients ${ }^{12}$.

The present study was done to evaluate the incidence of DVT and the factors that were correlated to DVT in elderly patients with proximal femur fracture who underwent surgery in our institution.

\section{MATERIALS AND METHODS}

This study was conducted on a consecutive series of patients with the age of 60 years old and older with proximal femur fracture (fractures of femoral head, femoral neck, or trochanteric) who underwent open reduction and internal fixation or hemiarthroplasty in our hospital. Patients with conservative treatment, or those who declined radiology or laboratory examination and those in whom contrast venography was contraindicated were excluded from the study. After taking consent from those eligible patients, daily observation for the clinical signs of DVT was carried out post-operatively, including Homan's sign, pain, oedema and skin discoloration of the lower extremity until the patients were discharged from the hospital. D-Dimer test was carried out on Day 2 after surgery. Between Day 4 and Day 7, contrast venography was carried out using iohexol $50 \mathrm{cc}$ as the contrast media on the extremity which had undergone surgery with the interpretation of results, and for detection of obstruction. Statistical analysis was done using Pearson chi square test to ascertain the relationship of D-Dimer and duration of surgery with the occurence of thrombosis. Fisher exact test was done to determine the correlation of clinical symptoms with the occurence of thrombosis because one of the values in the table is less than 5 .

\section{RESULTS}

There were 42 eligible patients in this study, $81 \%$ female, with the mean age in all subjects being 68.3 years old.
Eighteen of the 42 subjects ( $42.8 \%$ ) in this study were diagnosed as having DVT based on the result of contrast venography. There was an increased risk for DVT in those who had higher D-Dimer test, longer duration of surgery, and presence of clinical symptoms in the occurence of thrombosis $(1.40 ; 1.86 ; 1.09)$, although they were not statistically dignificant $(\mathrm{p}>0.05)$ as shown in Table II.

There were four patients who passed away due to complications of DVT. Two patients -passed away due to respiratory distress suspected to be due to pulmonary embolism within two weeks after operation. The other two passed away due to non-haemorrhagic stroke and acute myocardial infarct within two months and 4 months after surgery respectively.

\section{DISCUSSION}

The incidence of DVT among elderly people with proximal femur fracture in this study was $42.8 \%$ and this was similar in other study with Asian and Western population ${ }^{3}$. The pathogenesis of DVT is multifactorial and was described by Virchow due to three main factors known as Virchow's triad: hypercoagulability, endothelial injury, and venous stasis ${ }^{13}$. In proximal femur fractures which underwent surgery, endothelial injury can occur due to direct trauma or intraoperative mechanical factors. Postoperatively, the patients were immobilized and they experienced oedema that can lead to stasis of venous blood flow. Longer duration of surgery may contribute to prolonged venous stasis and increase the risk of endothelial injury of the vessel due to operative procedures. The result showed that patients who underwent surgery with duration of more than two hours was 1.86 times more susceptible to develop DVT as compared with patients who underwent surgery less than two hours, although statistically not significant. The other factor that may contribute to DVT in proximal femur fracture is the instrumentation of medullary canal during arthroplasty procedurs through -activation of the clotting cascade ${ }^{14}$. The combination of these factors raised the incidence of DVT in proximal femur fracture patients who underwent surgery. 
This study showed higher mortality rate $(22 \%$ within four months after surgery) as compared to previously reported study (20\% within 1 year after diagnosis is made) and this might be because of elderly population in this study ${ }^{1}$. Among the subjects with DVT, two passed away with pulmonary embolism. Another study showed that the incidence of pulmonary embolism cases following DVT in a lower extremity fractures was $15 \%-20 \%{ }^{15}$. There was one of fatal complication following DVT due to obstruction of the pulmonary arteries leading to various physiological changes ${ }^{16}$.

Most of the DVT cases were usually clinically silent and unpredictable, the symptomatic events being merely the tip of the iceberg ${ }^{17}$. This study also showed the same phenomenon, with about $77 \%$ of the subjects with DVT remaining asymptomatic. Plasma D-Dimers are produced when a mass of fibrin threads in a clotting process is degraded by plasmin, so the concentrations of these substances are raised in patients with DVT. It is activated by blood coagulation that is often promoted by inflammatory responses. However, in the elderly, the baseline of D-Dimer levels may be elevated due to the aging process ${ }^{12}$. It may affect the standard range of the clinical value of D-Dimer in this age group. Recommendation from various manufacturers of the D-Dimer assay is to determine the optimal cut off values for each population of patients who were tested ${ }^{18}$. High D-Dimer concentration is insufficient for making the diagnosis of DVT because it also occurs in other conditions such as malignancy, pregnancy and after operations8. In some VTE cases, it is used in conjunction with physical and other supporting examinations to reduce the need for imaging due to its high negative predictive value, the reason for which remains unclear ${ }^{19}$.
Clinical examination alone to diagnose DVT is often unreliable, and even additionally with negative D-Dimer test, the diagnosis of DVT still cannot be excluded. Therefore, further diagnostic procedures should be carried out for those with high index of suspicion to identify the occurence of thrombosis ${ }^{16,20}$.

Contrast venography can be done as one of the supporting examinations -due to its high sensitivity and specificity and can be considered -the gold standard for DVT ${ }^{21}$. When the thrombus is present, four cardinal signs, which are constant filling defect, abrupt termination of the dye column, nonfilling of the entire deep venous system or portion, and diversion of flow can be seen $^{22}$. In diagnosing DVT, contrast venography is performed when duplex ultrasound cannot be done or is technically not feasible, and in instances when the result of duplex ultrasound is negative but there is high clinical suspicion of the presence of thrombus. Relative contraindications are if patients have cellulitis at sites of venous access, allergy to contrast media, or in renal insufficiency patients who are not on dialysis. However, there are no absolute contraindications for contrast venography ${ }^{11}$.

In this study, the comorbidity aspects of the patients were not taken into account; further study that includes the evaluation of such variables is needed.

\section{CONCLUSION}

The incidence of DVT determined by contrast venography in elderly patients with proximal femur fracture who underwent surgery in our institution was relatively high (42.86\%), with the mortality rate due to complications of DVT at $22 \%$. High D-Dimer levels, longer duration of surgery, and presence of clinical symptoms increased the risk for DVT, although it was not statistically significant in our study. 


\section{REFERENCES}

1. Flinterman LE, Vlieg AH, Cannegieter SC, Rosendaal FR. Long-Term Survival in a large cohort of patients with venous thrombosis: incidence and predictors. PloS Medicine 2012; 9: 34-40.

2. Cheng G, Chan C, Liu YT, Choy YF, Wong MM, Yeung PKE, et al, Incidence of Deep Vein Thrombosis in Hospitalized Chinese Medical Patients and the impact of DVT prophylaxis. Hindawi Publishing Thrombosis. 2011; 2: 9-14.

3. Piovella F, Wang CJ, Lu H, Lee K, Lee LH, Lee WC, et al, Deep-vein thrombosis after major orthopaedic surgery in Asia. An epidemiological study based on postoperative screening with centrally adjudicated bilateral venography. Journal of Thrombosisand Haemostasis, 2005; 3: 2664-70.

4. Medscape. Anticoagulation Therapy for Venous Thromboembolism. Medscape General Medicine. 2004; 6: 3-5.

5. Vajanto I, Kuokkanen H, Niskanen R, Haapala J, Korkala O, Complications after treatment of proximal femoral fractures. Ann Chir Gynaecol 1998; 87(1): 49-52.

6. Ishimaru D, Ogawa H, Maeda M, Shimizu K. Outcomes of Elderly Patients with proximal femoral fractures according to positive criteria for surgical treatment. Orthop. 2012; 35(3): 353-8.

7. Abelseth G, Buckley RE, Pineo GE, Hull R, Rose MS, Incidence of deep-vein thrombosis in patients with fractures of the lower extremity distal to the hip. J Orthop Trauma. 1996; 10(4): 230-5.

8. Tovey C, Wyatt S, Diagnosis, investigationn, and management of deep vein thrombosis. BMJ 2003; 326: 1180-4.

9. Scarvelis D, Wells PS. Diagnosis and treatment of deep-vein thrombosis. CMAJ 2006; 175(9): 1087-92.

10. Terao M, Ozaki T, Sato T, Diagnosis of deep vein thrombosis after operation for fracture of the proximal femur: comparative study of ultrasonography and venography. J Orthop Sci. 2006 ;11(2): 146-53.

11. ACR-SIR Practice Guideline for the Performance of diagnostic infusion venography. The American College of Radiology 2013; 10: 417-24.

12. Adam SS., Key NS., Greenberg CS., D-Dimer antigen: current concepts and future prospects. Blood, 26 March 2009; 113(13): 2887-87.

13. Virchow R, Never fall von todlicher emboli der kungerarteries. Arch Path Anat 1856; 10: 225.

14. Sharrock NE, Go G, Harpel PC, Ranawat CS, The John Charnley Award: Thrombogenesis during -during total hip arthroplasty. Clin Orthop 1995; 319: 16-27.

15. Kucher N. Clinical practice. Deep-vein thrombosis of the upper extremities. N Engl J Med 2011; 364: 861-9.

16. Bortholomew JR., Venous Thromboembolism (Deep venous thrombosis \& pulmonary embolism). Cleveland Clinis Medical Pub 2004; 21-31.

17. Clagett PG, Anderson FA, Heit JA, Lieberman JR, Brownell H, Prevention of venous thromboembolism. Chest 1998, 114(S): 531-605.

18. Legnani C, Palareti G, Cosmi B, Cini M, Tosetto A, Tripodi A, et al. Different cut-off values of quantitative D-dimer methods to predict the risk of venous thromboembolism recurrence: a post-hoc analysis of the PROLONG study. Haematologica. 2008; 93 : 900-7.

19. Aschwanden M, Labs KH, Jeanneret C, Gehrig A, Jaeger KA. The value of rapid D-dimer testing combined with structured clinical evaluation for the diagnosis of deep vein thrombosis. J Vasc Surg 1999; 30: 929-35.

20. Wells PS, Anderson DR, Rodger M, Forgie M, Kearon C, Dreyer J, Evaluation of D-Dimer in the Diagnosis of Suspected Deep Vein Thrombosis. N Engl J Med 349: 13.

21. Rossi R., Agnelli G., Current role of venography in the diagnosis of deep-vein thrombosis. Minerva Cardioangiol 1998; 46(12): 507-14.

22. Robinov K, Paulin S. Roentgen diagnosis of venous thrombosis in the leg. Arch Surg. 1972; 104: 134-44. 